\title{
Early TIPS for portal hypertensive related bleeding: Is resource or education the reason for failure to show clear survival benefit?
}

\author{
Peter Hayes ${ }^{1}$, Rajeshwar P Mookerjee ${ }^{2, *}$
}

${ }^{1}$ Division of Health Sciences, Royal Infirmary of Edinburgh, Queens Medical research Institute, Edinburgh, UK; ${ }^{2}$ University College London Institute of Liver and Digestive Health, UCL Medical School, Royal Free Hospital, Rowland Hill Street, London, UK

${ }^{*}$ Corresponding author. Address: University College London Institute of Liver and

Digestive Health, UCL Medical School, Royal Free Hospital, Rowland Hill Street, London, UK. Tel.: +44 2074332853. Email address: r.mookerjee@ucl.ac.uk (R. Mookerjee) 
In this issue of the Journal, Thabut and colleagues embark on a large multicenter audit in France, wherein they analyze the application of early transjugular intrahepatic portosystemic shunt (TIPS), defined as intervention within 72 hours of the index endoscopy, for portal hypertension (PHT) related bleeding. [1] Their study demonstrates that of $35 \%$ of patients meeting current eligibility criteria for early TIPS (namely, Child C cirrhosis $<13$ points or Child B with active bleeding at endoscopy)[2], in 'real life', barely $7 \%$ of patients received this intervention. Furthermore, in these limited numbers of patients receiving early TIPS, most had less advanced disease and therefore a lower risk of bleeding mortality. Whilst the lack of technical availability was mooted as a potential reason, it transpires that over one-third of centers had daily feasibility to perform TIPS, although feasibility and actually being capable of delivering are two separate issues. Consequently, it is perhaps unsurprising that on multivariate analysis, early TIPS failed to show any proven benefit in this population and the only independent risk factor associated with survival in this study was severity of liver disease.

In a recent meta-analysis of four studies that were designed specifically to address the role of early TIPS,[3] two studies, both randomised, showed a significant increase in survival, albeit, neither was designed to assess survival as a primary endpoint.[2, 4] The other two studies did not find a statistically significant increase in survival, with one including patients with Child-Pugh score to 15 points and thereby, very advanced disease.[5, 6$]$ The overall finding of the meta-analysis did suggest that early TIPS increased control of bleeding, decreased rate of re-bleeding and did increase survival. This was further supported by a recent large retrospective study from the US, where early TIPS improved mortality and reduced re-bleeding with no 
appreciable increase in the incidence of hepatic encephalopathy, and a similar retrospective European study.[7, 8]

One of the features of PHT-related bleeding is the relationship with a systemic inflammatory response and increased gut bacterial translocation, which may further aggravate organ dysfunction and precipitate the onset of acute-on-chronic liver failure.[9] In this regard, a potential explanation for reduced mortality after TIPS is the immediate reduction in portal hypertension and associated pathophysiology, which facilitates reduced gut bacterial translocation and the related systemic inflammatory response. This has been demonstrated by data showing a reduction in endotoxin and TNF receptor levels post elective TIPS [8], as well as reduced levels of chemokines such as CXCL9.[10,11]

A potential weakness in the study described by Thabut and colleagues[1] is that no data was recorded on other morbidity factors, including hepatic encephalopathy, or progression of liver decompensation, nor re-bleeding events. It would have been interesting to note post-TIPS, whether the model for end-stage liver disease (MELD) score or level of hepatic decompensation progressed further in these patients longer term. Also of note is that in patients with refractory bleeding, six-week mortality was $18 \%$ compared to $9 \%$ in the early TIPS group $(p=0.32)$, and refractory bleed patients had much higher baseline Child-Pugh (10.7 vs. 8.9) and MELD (20 vs. 16) scores, and presentation with encephalopathy ( $45 \%$ vs. $15 \%)$. This underlies the limitation of use of Child-Pugh or MELD scores alone to define the 'high-risk' group who may have the biggest mortality benefit from early TIPS, in part, since some of their components such as ascites and creatinine may improve post-TIPS, whilst other factors such as bilirubin and encephalopathy, may progress. Indeed, liver failure after TIPS may occur in up to $10 \%$ cases, despite an MELD <12. [12] 
A seminal randomised study by Monescillo et al. demonstrated that in 116 patients in whom the hepatic venous pressure gradient (HVPG) was performed within 24 hours of an acute variceal bleed, failure to control bleeding and one-year mortality were associated with HVPG $>20 \mathrm{mmHg}$, and that early TIPS reduced treatment failure and mortality. [4] This suggested that haemodynamic factors may define a high-risk group, though clearly HVPG measurement during an index bleed is impractical. It remains to be established whether other surrogates of portal hypertension, such as Fibroscan or real-time shear wave technology, or even platelet count, can be used to predict hepatic dysfunction and optimal candidates for early TIPS. [13] Similarly, it is possible that reduced markers of systemic inflammatory response may predict beneficial response and survival after TIPS, as has been suggested for the chemokine, CXCL11.[14].

The study reported by Thabut et al. shows the mean delay to TIPS placement was $1.9 \pm 0.8$ days, albeit the median delay till TIPS was $3.4(2.6-4.4)$ days. This could suggest that the apparent control of bleeding at index endoscopy makes physicians reluctant to pre-emptively use TIPS in patients within 72 hours of the index bleed. If so, what might be their concern? A lack of confidence in current data on improved control of bleeding and survival may be inferred from this study, though other concerns may exist on precipitation of hepatic encephalopathy or worsening of liver function, in patients in whom bleeding is controlled. Of note, at the start of the audit only eight centers included early TIPS in their local bleeding guidelines, despite the then Baveno $V$ guidelines [15]; a reflective questionnaire as a follow on to this audit showed that $<15 \%$ changed their practice despite the subsequent Baveno VI recommendations, and as a refection of this, there was no increase in TIPS service provision.[16] Furthermore, most responders continued to hold an opinion that evidence for early 
TIPS remained weak, as shown by the mean questionnaire response addressing this question of 4.43 out of a linear scale from 1 to 10 .

Thabut et al.[1] also found that many of the non-academic centers used considerably fewer vaso-active drugs, perhaps suggesting sub-optimal medical therapy was applied before a decision on TIPS requirement was undertaken. This would also influence the requirement for TIPS, if based on endoscopic signs of active bleeding at first endoscopy, even if less severe liver disease was present. Indeed, we learn that there was a higher proportion of active bleeding at endoscopy in nonacademic centers. This observation was further compounded by the limited use of antibiotics, particularly in the non-academic centers (40\% did not receive antibiotics at the index bleed), and in the knowledge that the outcome was worse in those with infection at the outset. This reflects the 'real world' nature of such studies where despite the presence of existing guidelines, variations in clinical practice may bias the primary outcome away from the applied intervention - in this case outcome of early TIPS.

An area of on-going debate exists as to the cut-off for liver disease severity (most often capped at Child-Pugh score C-13), where perceived benefit from early TIPS is perhaps out-weighed by poor survival in those with advanced disease.[6] This is thought to relate to impaired liver perfusion after TIPS and the associated risk of liver failure in such decompensated patients. In decompensated patients, placement of TIPS may facilitate an opportunity to list and bridge patients, who had not previously been identified before an index bleed, to liver transplantation over a shorter time frame. Whilst it is clear that patients with more stable Child A and B cirrhosis generally have good outcomes, as seen by Thabut and colleagues, further randomised studies are clearly needed, specifically in Child C patients, to assess whether there is a ceiling of severity at which TIPS fails to improve outcomes. 
In summary, the study by Thabut et al. in this issue of the Journal highlights the 'real life' difficulties in adherence to clinical guidelines such as Baveno VI, when physician bias may preclude deployment of an intervention, that indeed may have the potential to significantly improve outcomes in PHT-related bleeding. More prospective randomised studies demonstrating the benefit of early TIPS will undoubtedly help improve adherence to bleeding guidelines. This needs to be coupled with better access to out-of-hours TIPS services.

\section{Financial support [H1]}

The authors received no financial support for the production of this manuscript.

\section{Conflicts of interest [H1]}

The authors declare no conflicts of interest that pertain to this work.

\section{Authors' contributions [H1]}

RPM and PH contributed equally to this work

\section{References}

[1] Thabut D, Pauwels A, Carbonell N, Remy AJ, Nahon P, Causse X, et al. Cirrhotic patients with portal hypertension-related bleeding and an indication for early-TIPS: a large multicentre audit with real-life results. J Hepatol 2017. 
[2] Garcia-Pagan JC, Caca K, Bureau C, Laleman W, Appenrodt B, Luca A, et al. Early use of TIPS in patients with cirrhosis and variceal bleeding. N Engl J Med 2010;362:2370-2379.

[3] Deltenre P, Trepo E, Rudler M, Monescillo A, Fraga M, Denys A, et al. Early transjugular intrahepatic portosystemic shunt in cirrhotic patients with acute variceal bleeding: a systematic review and meta-analysis of controlled trials. Eur $\mathrm{J}$ Gastroenterol Hepatol 2015;27:e1-9.

[4] Monescillo A, Martinez-Lagares F, Ruiz-del-Arbol L, Sierra A, Guevara C, Jimenez E, et al. Influence of portal hypertension and its early decompression by TIPS placement on the outcome of variceal bleeding. Hepatology 2004;40:793-801. [5] Garcia-Pagan JC, Di Pascoli M, Caca K, Laleman W, Bureau C, Appenrodt B, et al. Use of early-TIPS for high-risk variceal bleeding: results of a post-RCT surveillance study. J Hepatol 2013;58:45-50.

[6] Rudler M, Cluzel P, Corvec TL, Benosman H, Rousseau G, Poynard T, et al. Early-TIPSS placement prevents rebleeding in high-risk patients with variceal bleeding, without improving survival. Aliment Pharmacol Ther 2014;40:1074-1080. [7] Njei B, McCarty TR, Laine L. Early transjugular intrahepatic portosystemic shunt in US patients hospitalized with acute esophageal variceal bleeding. $\mathrm{J}$ Gastroenterol Hepatol 2017;32:852-858.

[8] Bucsics T, Schoder M, Goeschl N, Schwabl P, Mandorfer M, Diermayr M, et al. Re-bleeding rates and survival after early transjugular intrahepatic portosystemic shunt (TIPS) in clinical practice. Dig Liver Dis 2017.

[9] Mookerjee RP. Acute-on-chronic liver failure: the liver and portal haemodynamics. Curr Opin Crit Care 2011;17:170-176. 
[10] Trebicka J, Krag A, Gansweid S, Appenrodt B, Schiedermaier P, Sauerbruch $\mathrm{T}$, et al. Endotoxin and tumor necrosis factor-receptor levels in portal and hepatic vein of patients with alcoholic liver cirrhosis receiving elective transjugular intrahepatic portosystemic shunt. Eur J Gastroenterol Hepatol 2011;23:1218-1225. [11] Berres ML, Asmacher S, Lehmann J, Jansen C, Gortzen J, Klein S, et al. CXCL9 is a prognostic marker in patients with liver cirrhosis receiving transjugular intrahepatic portosystemic shunt. J Hepatol 2015;62:332-339.

[12] Luca A, Miraglia R, Maruzzelli L, D'Amico M, Tuzzolino F. Early Liver Failure after Transjugular Intrahepatic Portosystemic Shunt in Patients with Cirrhosis with Model for End-Stage Liver Disease Score of 12 or Less: Incidence, Outcome, and Prognostic Factors. Radiology 2016;280:622-629.

[13] Bureau C, Metivier S, D'Amico M, Peron JM, Otal P, Pagan JC, et al. Serum bilirubin and platelet count: a simple predictive model for survival in patients with refractory ascites treated by TIPS. J Hepatol 2011;54:901-907.

[14] Berres ML, Lehmann J, Jansen C, Gortzen J, Meyer C, Thomas D, et al. Chemokine (C-X-C motif) ligand 11 levels predict survival in cirrhotic patients with transjugular intrahepatic portosystemic shunt. Liver Int 2016;36:386-394.

[15] de Franchis R, Baveno VF. Revising consensus in portal hypertension: report of the Baveno $\mathrm{V}$ consensus workshop on methodology of diagnosis and therapy in portal hypertension. J Hepatol 2010;53:762-768.

[16] de Franchis R, Baveno VIF. Expanding consensus in portal hypertension: Report of the Baveno VI Consensus Workshop: Stratifying risk and individualizing care for portal hypertension. J Hepatol 2015;63:743-752. 